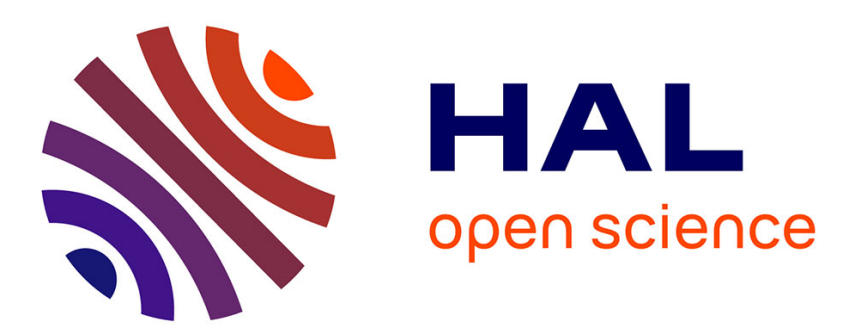

\title{
Journalistes et revues féministes anarchistes en Italie au début du XXe siècle
}

\author{
Laura Fournier-Finocchiaro
}

\section{To cite this version:}

Laura Fournier-Finocchiaro. Journalistes et revues féministes anarchistes en Italie au début du XXe siècle. Sens public, 2021, Des journaux pour toutes. Femmes et féministes dans la presse en France, Italie et Espagne au XXe siècle. hal-03352843

\section{HAL Id: hal-03352843 \\ https://hal.science/hal-03352843}

Submitted on 23 Sep 2021

HAL is a multi-disciplinary open access archive for the deposit and dissemination of scientific research documents, whether they are published or not. The documents may come from teaching and research institutions in France or abroad, or from public or private research centers.
L'archive ouverte pluridisciplinaire HAL, est destinée au dépôt et à la diffusion de documents scientifiques de niveau recherche, publiés ou non, émanant des établissements d'enseignement et de recherche français ou étrangers, des laboratoires publics ou privés. 


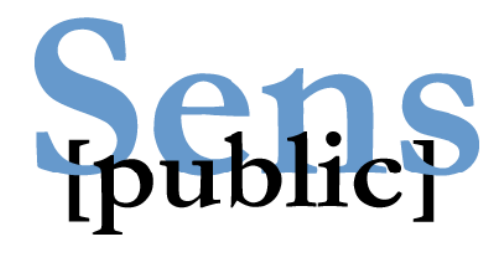

\title{
Journalistes et revues féministes anarchistes en Italie au début du XXe siècle
}

\author{
Laura Fournier-Finocchiaro
}

Publié le 01-07-2021

http://sens-public.org/articles/1586

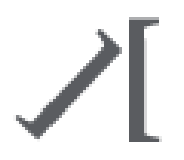

Creative Commons Attribution-NonCommercial-ShareAlike 4.0

International (CC BY-NC-SA 4.0) 


\title{
Résumé
}

Après un bref aperçu de la participation des femmes écrivaines à la presse anarchiste en Italie au début du $\mathrm{XX}^{\wedge} \mathrm{e}^{-}$siècle, l'article examine les grandes idées développées dans les premières revues féminines anarchistes publiés en Italie, La Donna libertaria (1912-1913) et L'Alba libertaria (1915). Outre à inciter les femmes à participer à la lutte anticapitaliste et antimilitariste, les revues féminines anarchistes montrent une certaine ouverture aux problèmes des femmes, en formulant notamment des prises de position originales sur la maternité et le contrôle des naissances par la contraception. Les revues accusent en même temps les compagnons anarchistes d'avoir négligé l'élément féminin au sein du mouvement et entendent lutter contre l'antiféminisme de tous bords.

\begin{abstract}
After a brief overview of the participation of female writers in the anarchist press in Italy at the beginning of the twentieth century, the article examines the main ideas developed in the first anarchist women's periodicals published in Italy, La Donna libertaria (19121913) and L'Alba libertaria (1915). In addition to encouraging women to take part in the anti-capitalist and anti-militarist struggle, anarchist women's periodicals showed some openness to women's issues, in particular by formulating original positions on motherhood and birth control through contraception. At the same time, the periodicals accuse the anarchist companions of having neglected the female element within the movement and intend to fight against antifeminism of all kinds.
\end{abstract}

Mot-clés : Anarchisme, Presse anarchiste, Antimilitarisme, Maternité, Féminisme, La Donna libertaria, L'Alba libertaria, Leda Rafanelli, Nella Giacomelli, Aida Latini

Keywords: Anarchism, Anarchist Press, Antimilitarism, Maternity, Feminism, La Donna libertaria, L'Alba libertaria, Leda Rafanelli, Nella Giacomelli, Aida Latini 


\section{Table des matières}

Femmes écrivaines dans la presse anarchiste . . . . . . . . . . 5

La Donna libertaria et les combats anarchistes . . . . . . . . . . . . 11

Liberté de maternité et rôle des mères . . . . . . . . . . . . . . . . 14

La lutte contre l'antiféminisme . . . . . . . . . . . . . . . . . . . . . . . . . . . .

Bibliographie ................... . . . 21 


\title{
Journalistes et revues féministes anarchistes en Italie au début du XXe siècle
}

\author{
Laura Fournier-Finocchiaro
}

À partir des dernières décennies du XIX ${ }^{\mathrm{e}}$ siècle, la présence féminine s'intensifia considérablement dans le panorama culturel et littéraire italien. Les publications d'écrits féminins connaissent une augmentation exponentielle (Corabi 2012) et on retrouve des traces de discours féministes non seulement dans la presse féminine politique favorable au vote féminin, mais aussi dans les périodiques modérés et dans la presse généraliste (Buttafuoco 1988; Pisano 2004 ; Gazzetta 2018). Au sein du mouvement ouvrier, presque chaque feuille périodique rédigée par des associations ou groupes politiques contient une rubrique ou des pages entières consacrées aux travailleuses, où s'expriment des écrivaines et journalistes, qui souvent se connaissaient entre elles et tissaient des réseaux qui traversaient la péninsule. Même les socialistes des classes moyennes faisaient partie de cette nébuleuse féministe, qui se retrouva lors du premier Congrès féminin à Rome au printemps 1908 (Frattini 2008). De la même façon, un nombre non négligeable de femmes participa à la presse périodique des mouvements anarchistes italiens, même si elles sont souvent ignorées dans les recherches historiques sur le premier féminisme - à l'exception de Cantarelli (1980) et Bignami (2011, 2016, 2018) -, tout comme les premiers mensuels féminins anarchistes publiés en Italie, La Donna libertaria, qui voit le jour en 1912, et L'Alba libertaria, qui publie quatre numéros en 1915 .

Dans cet article, nous donnerons d'abord un bref aperçu de la participation féminine à la presse anarchiste en Italie au début du $\mathrm{XX}^{\mathrm{e}}$ siècle et des combats menés souvent en couple par les femmes écrivaines. Nous examinerons ensuite les grandes idées développées dans les revues féminines anarchistes, non seulement leur contribution à la propagande anarchiste et antimilita- 
Journalistes et revues féministes anarchistes en Italie au début du XXe siècle

riste, mais aussi leur ouverture aux problèmes des femmes, en particulier la maternité et la lutte contre l'antiféminisme.

\section{Femmes écrivaines dans la presse anarchiste}

Dans les milieux anarchistes, le militantisme est souvent vécu en couple ou en famille, même si tous les anarchistes n'encouragent pas forcément la participation féminine à la presse et ne s'engagent pas pour défendre l'émancipation des femmes. En Italie comme ailleurs, l'anarchisme est caractérisé par la «prolifération des tendances » (Antonioli et Masini 1999; Berti 2003; Giulietti 2012) : communiste, pédagogue, syndicaliste, individualiste, antimilitariste, néomalthusien... En particulier, l'impérialisme des gouvernements européens donne forme à un groupe d'opposants internationalistes et anticolonialistes qui organise des manifestations et contribue à la multiplication de la propagande écrite antimilitariste, à laquelle participent aussi des femmes (Scriboni 2008; Bignami 2016; Bartoloni 2017).

Parmi les couples de militants qui donnent vie à des titres de presse importants, on peut citer celui formé par Pasquale et Zelmira Binazzi ${ }^{1}$, qui fonde à La Spezia l'hebdomadaire Il Libertario (1903-1922), un des périodiques anarchistes les plus populaires de la péninsule. Zelmira Binazzi contribue à la diffusion du journal et signe de nombreux articles, principalement à caractère culturel, ainsi qu'une série de poèmes. Elle remplace son mari à plusieurs reprises dans la direction et l'administration de l'hebdomadaire, notamment lorsqu'il est engagé dans des tournées de conférences en Italie et à l'étranger, ou lorsqu'il est emprisonné. Elle partage avec lui les hauts et les bas de la vie du Libertario, marquée par les censures, les séquestres, les procès et condamnations, même au prix de sa propre liberté. À Milan, le militant anarchiste Giovanni Gavilli lance le journal Il Grido della folla (1902-1905) avec la collaboration de sa première compagne, Aida Latini ${ }^{2}$ et de Nella Gia-

1. Zelmira Binazzi (1865-1936), née Carlotta Germinia Peroni dans la région de la Lunigiana, épouse Pasquale Binazzi en 1901 (Antonioli 2003).

2. Aida Latini (1882-1932) participa à toutes les manifestations des partis subversifs, ce qui lui valut d'être arrêtée et emprisonnée en 1910 (Bignami 2016,63). Elle se lie après la guerre avec Giovanni Romiti, anarchiste de Pontremoli, mais elle est ensuite soupçonnée d'être une espionne de la police. Le couple finira par adhérer au fascisme (Sacchetti 1983, $77)$. 
Journalistes et revues féministes anarchistes en Italie au début du XXe siècle

comelli (nom de plume Iréos) ${ }^{3}$. Giacomelli, en tandem avec Ettore Molinari (nom de plume Epifane) ${ }^{4}$, publie notamment des textes de théorisation du 《 libre amour » (Giacomelli 1903a, 1903b) et fait partie des premières journalistes à dénoncer les préjugés des anarchistes envers l'émancipation de leurs compagnes (Giacomelli 1902).

La maîtresse d'école Fanny Dal Ry ${ }^{5}$ et la jeune Leda Rafanelli, certainement l'anarchiste la plus connue du $\mathrm{XX}^{\mathrm{e}}$ siècle ${ }^{6}$, participent quant à elles aux journaux anarchistes Il Pensiero (1903-1911), publié à Rome par Pietro Gori et Luigi Fabbri, et à La Pace, periodico quindicinale antimilitarista, fondé à Gênes en 1903 et dirigé par le jeune étudiant socialiste révolutionnaire d'origine toscane Ezio Bartalini (Dal Ry 1920; Giacomini 1990). Leda Rafanelli emboîte le pas de Nella Giacomelli pour y défendre la nécessité du féminisme (Rafanelli 1904). Dans La Pace, puis dans le journal antimilitariste Energia!, fondé en 1905 par des jeunes socialistes napolitains, elle publie aussi des odes, des récits et des articles contre la guerre. Son objectif est polémique et éducatif : elle invite à prendre position contre la conscription obligatoire, considérée comme une manifestation de la violence de l'État, et cherche à acquérir les femmes à la cause antimilitariste (Rafanelli 1906). Pour Elena Bignami, on assiste à " une sorte de "féminisation" de cette campagne, menée ad hoc par les anarchistes dans une double direction qui voit la femme à la fois objet et sujet de l'action militante »(Bignami 2016, 61).

Rafanelli et son mari Luigi Polli poursuivent leur propagande dans la revue $L a$ Blouse, fondée par Polli et publiée à Florence, tandis qu'à Milan un autre couple d'anarchistes, Filippo Corridoni et Maria Rygier (Montesi 2013), donnent naissance au bimensuel antimilitariste Rompete le file!, qui publie une dizaine de numéros en 1907. Maria Rygier, qui en 1906 avait épousé Virgi-

3. Nella Giacomelli (1873-1949) s'affirme comme la voix de l'intransigeance anarchiste et de l'internationalisme le plus rigoureux (Masini 1973; Ongaro 2019).

4. Chimiste anarchiste, Ettore Molinari avait engagé en 1900 Giacomelli comme gouvernante de ses enfants.

5. Fanny Dal Ry (1877-1961) vécut en concubinage avec Bartalini jusqu'à la Grande guerre. Elle publia plus d'une trentaine d'articles dans La Pace (Mangani 1989; Tagliaventi 2014).

6. Le parcours original de Leda Rafanelli (1880-1971), qui l'amène à fréquenter le jeune Mussolini et les futuristes de gauche, marqué par l'amour pour la culture arabo-musulmane et sa conversion à l'Islam, a même fait l'objet d'une bande dessinée (Colaone, Satta, et De Santis 2016). Pour une vision d'ensemble du personnage, voir Chessa (2008); et en français, Guidoni (1994); Mauri (2011). 
Journalistes et revues féministes anarchistes en Italie au début du XXe siècle

nio Corradi et avait fait de leur maison milanaise un centre d'activité révolutionnaire, est rejointe par Aida Latini et Nella Giacomelli. Suite à ses articles et à sa participation répétée à des manifestions anti-gouvernementales, Rygier est condamnée en 1908 à plus de cinq ans de prison, jetant les bases de la mythification de sa personnalité, qui devient un objet de profonde admiration pour ses compagnons et un nouvel instrument de propagande antimilitariste. En effet, l'image de l'agitatrice en tenue de prisonnière, diffusée sous forme de carte postale par la Fédération socialiste milanaise à l'occasion du premier mai 1908, sanctionna sa désignation comme héroïne de l'anarchisme. Le journal Il Libertario lança une campagne pour la libération de Maria Rygier, désignée comme « la vestale de la paix universelle ». 
Journalistes et revues féministes anarchistes en Italie au début du XXe siècle

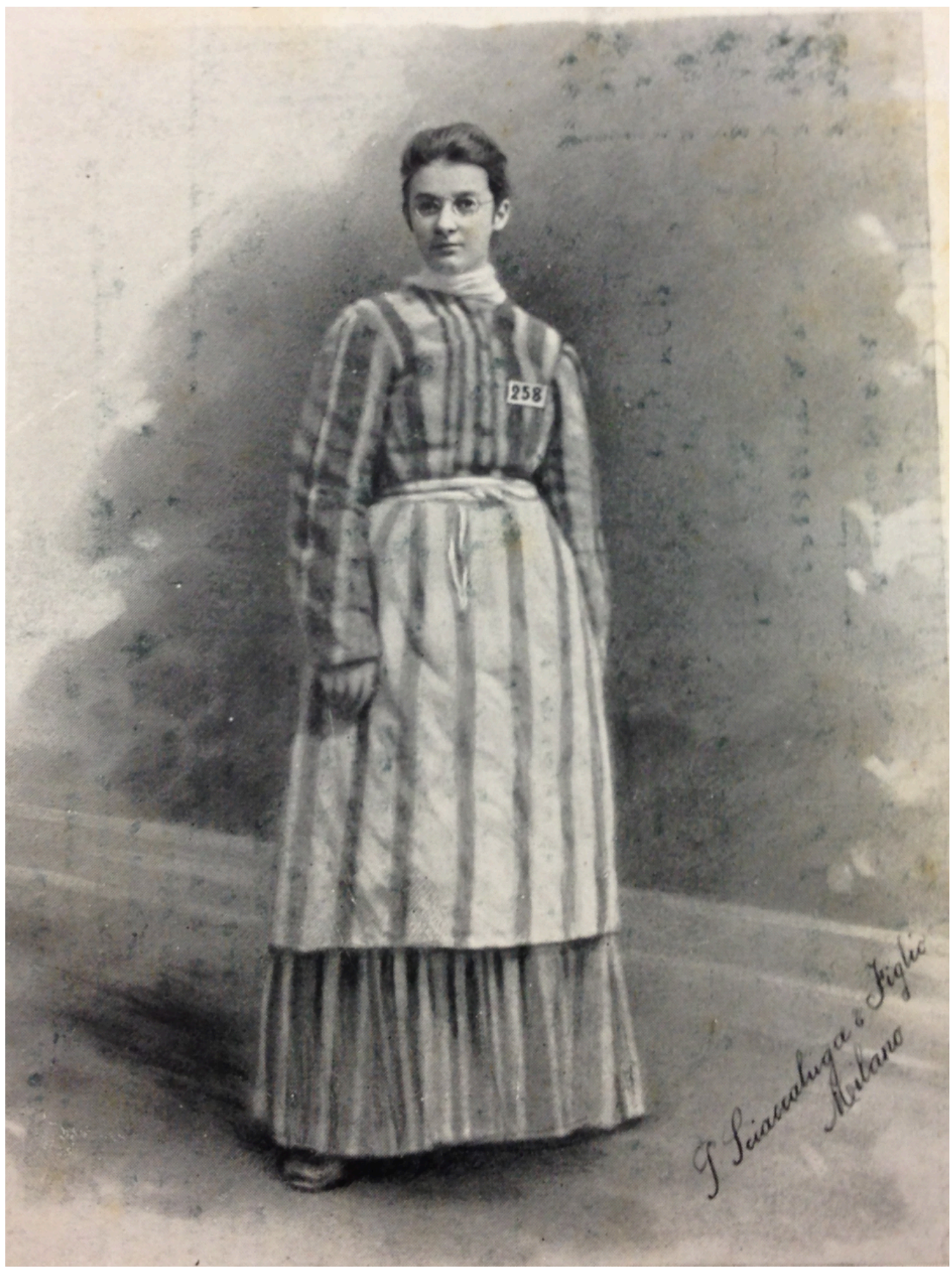

Figure 1 - Carte postale avec le portrait de Maria Rygier en tenue de prisonnière, mise en vente par la Federazione Socialista Milanese à l'occasion du 1er mai 1908 (Collection privée). 8 
Journalistes et revues féministes anarchistes en Italie au début du XXe siècle

Giacomelli et Molinari font appel à Leda Rafanelli et à son nouveau compagnon Giuseppe Monanni pour collaborer à un autre journal publié à Milan, La Protesta umana (1906-1909), qui se fait le porte-parole du courant anarchiste individualiste, farouchement opposé à la violence et à la guerre. La Protesta umana publie aussi des articles de Francesca Aondio ${ }^{7}$, qui s'adresse directement aux femmes et accuse ses compagnons anarchistes d'avoir négligé l'élément féminin (Aondio 1907, 1909); un autre article pointe la déficience de l'action de propagande libertaire tant que le sexe féminin, « qui représente en soi une force formidable », restera abandonné (M. 1907).

La guerre en Libye déclenche une accélération de la propagande et de l'action antimilitariste anarchiste et mobilise les femmes journalistes. Par exemple, l'hebdomadaire de Pise L'Avvenire anarchico (1910-1922) consacre une rubrique, Palestra femminile, aux voix féminines de l'anarchisme, dirigée par Priscilla Poggi ${ }^{8}$ et sa fille Jessa Fontana ${ }^{9}$, à laquelle participent Rafanelli et Giacomelli. Cette ouverture permet d'enrichir la propagande anarchiste de nouveaux thèmes, tout en compliquant par de nouveaux points de vue la dialectique libertaire. Poggi, en particulier, pointe la responsabilité des mères dans l'échec de l'antimilitarisme, pour leur passivité face aux sirènes du patriotisme. Entre temps, Maria Rygier est renvoyée en prison pour avoir pris la défense de l'anarchiste Augusto Masetti, auteur de l'homicide d'un colonel, et cette nouvelle détention revigore son aura mythique. Tandis qu'Aida Latini défend la valeur de l'icône de l'antimilitarisme dans ses conférences, le groupe féminin libertaire de Parme choisit le nom " Maria Rygier » en son honneur. Les militantes, pour concrétiser leur soutien à Rygier, donnent naissance au premier mensuel féminin anarchiste publié en Italie, La Donna libertaria, dont paraissent huit numéros entre octobre 1912 et juin $1913^{10}$.

7. Il existe peu d'informations sur Francesca Aondio, née en 1882 près de Côme et compagne de l'anarchiste toscan Arturo Pruneti, émigrée en Belgique puis à Calais (Bignami 2016, 75).

8. Priscilla Poggi (1861-1949) est une ouvrière textile, mariée à l'anarchiste Ettore Fontana, qui participe à toutes les manifestations organisées à Pise (Bignami 2016, 70-71).

9. Jessa Fontana (1883-1961) est la fille ainée du couple Fontana-Poggi et participe dès son plus jeune âge aux activités du mouvement anarchiste de Pise. En 1901 elle est condamnée à deux mois et demi de réclusion. En 1910 elle se marie avec Giuseppe Pieroni (Bignami 2016, 72).

10. La revue a été numérisée et mise en ligne par la Biblioteca Libertaria Armando Borghi. 
Journalistes et revues féministes anarchistes en Italie au début du XXe siècle

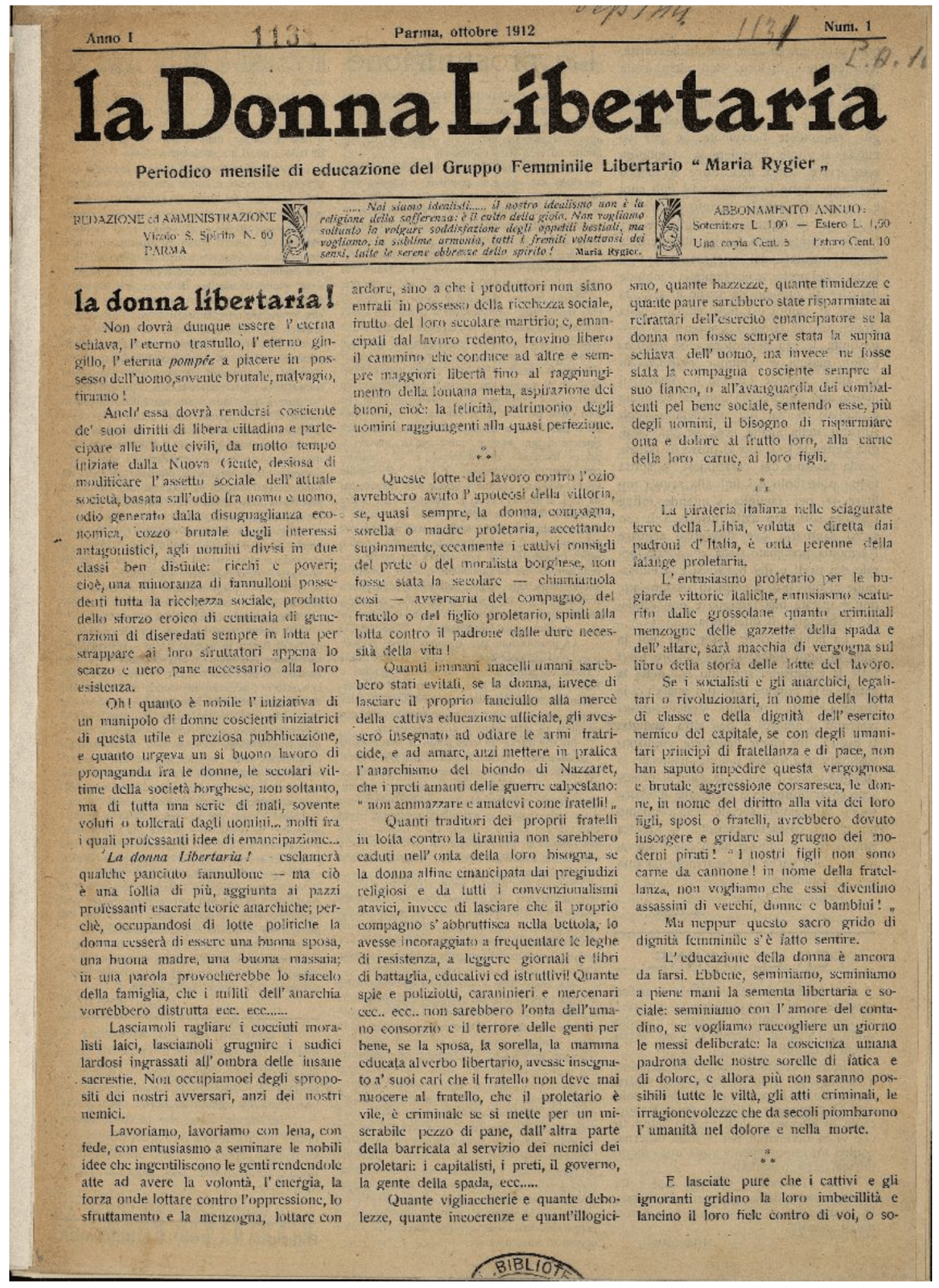

Figure 2 - Première de couverture de La Donna libertaria, Parma, ${ }^{\circ} 1$, octobre 1912 (Biblioteca Libertaria Armando Borghi, Castel Bolognese, Italie) 
Journalistes et revues féministes anarchistes en Italie au début du XXe siècle

\section{La Donna libertaria et les combats anarchistes}

Les trois premiers numéros de la revue, jusqu'en décembre 1912, sont publiés à Parme sous la direction d'Amelia Legati et la responsabilité éditoriale d'Antonio Melegari ${ }^{11}$. Elle porte comme sous-titre Periodico mensile di educazione del Gruppo femminile libertario "Maria Rygier ». En février 1913, le siège est transféré à Forlì et la revue, publiée jusqu'en juin avec le nouveau sous-titre Periodico mensile di propaganda educativa e libertaria femminile, passe sous la direction d'Adele Servisi, Marzia Rossi et Irma Guidaloni, et sous la responsabilité éditoriale d'Armando Sintoni ${ }^{12}$.

Cette revue est particulièrement mystérieuse, car l'identité réelle des rédactrices n'est pas connue, et aucun des pseudonymes n'est réemployé par la suite. Aucun des noms féminins qui servent à signer les articles, contrairement à ceux des autres femmes journalistes anarchistes, n'est par ailleurs fiché par les services de police du Royaume d'Italie (Casellario politico centrale). Comme nous le verrons plus loin, certains imaginèrent même qu'il s'agissait de journalistes masculins travestis en femmes, hypothèse démentie par les rédactrices. Il est possible que derrière certains des pseudonymes féminins puissent se cacher Aida Latini, Nella Giacomelli, Leda Rafanelli ou Priscilla Poggi Fontana, ou bien que les femmes de ce collectif n'aient pas trouvé, ni avant ni après, d'autres périodiques où s'exprimer. Parmi les rédacteurs, on remarque la signature de Giovanni Romiti, futur compagnon d'Aida Latini, leader des anarchistes de Pontremoli et futur gérant de L'Alba libertaria, et quelques pseudonymes masculins attribués, comme Eliseo Ruscello (alias Giuseppe Bacchini) et Eliseo Montagna (alias Mario Balestra) (Antonioli 2002).

L'article de présentation, signé Tomasina, annonce d'emblée l'objectif de la revue, qui est de faire un travail de propagande visant les femmes, " non

11. Antonio Melegari (né en 1891) est un mécanicien et décorateur anarchiste connu des autorités de Parme; il est également directeur, avec Cleto Evaristo Marcacci, du journal La Barricata de Parme (1912-1913), organe du Circolo libertario di studi sociali, qui publie aussi des articles de Leda Rafanelli (Bignami 2016, 73).

12. Armando Sintoni (né en 1890) est un activiste, membre de l'Unione anarchica forlivese et également gérant de L'Agitatore de Bologne. Il sera condamné pour « provocation au crime » à 5 mois de prison et 175 lires d'amende pour l'article publié dans le $\mathrm{n}^{\mathrm{o}} 4 \mathrm{du}$ journal, intitulé «1911-1912-1913 », qui accusait le gouvernement italien d'avoir envoyé à la mort les combattants dans les guerres coloniales ( $\mathrm{Il}$ nostro processo in tribunale » 1913, 1). 
Journalistes et revues féministes anarchistes en Italie au début du XXe siècle

seulement les victimes séculaires de la société bourgeoise, mais de toute une série de maux, souvent voulus ou tolérés par les hommes... parmi ceux qui professent des idées d'émancipation » (Tomasina 1912c). Selon l'historienne Rossella Cantarelli, la revue est surtout un moyen de diffusion de la pensée anarchiste auprès du public féminin : « une partie des articles du journal vise à exposer les canons de l'anarchie à l'élément féminin et notamment à réévaluer la figure des anarchistes, trop souvent et à tort considérés comme violents » (Cantarelli 1980, 240).

Ainsi Eliseo Ruscello (1912a, 1-2) explique « Qui sont les anarchistes?», et Tomasina (1912d, 2-3) répond à la question « Sommes-nous violents? ». Le journal privilégie le thème de l'antimilitarisme (Tomasina 1912c, 1-2; Legati 1913a, 2) et prend la défense des anarchistes persécutés par la justice, tout particulièrement Maria Rygier, figure exemplaire de victime politique de l'État. Rygier n'envoie cependant aucune contribution au journal; elle signe uniquement une lettre formelle de remerciements adressée à Amelia Legati qui lui avait fait part de son initiative (Rygier 1912, 3). Après sa sortie de prison en décembre 1912, l'ex-détenue poursuit sa campagne antimilitariste dans la revue L'Agitatore de Bologne, dont elle prend la direction. Cantarelli émet l'hypothèse que cette indifférence est peut être la cause du désengagement du groupe de Parme et du transfert de la revue à Forli, mais il est plus vraisemblable que le changement de responsable soit dû au caractère très souvent éphémère de cette charge, qui impliquait le risque d'être poursuivi et condamné par la justice.

Lorsque Amelia Legati (1913b, 1) passe le relais aux nouvelles directrices, elle présente le périodique comme « le premier féminin au monde », et réitère le caractère du journal, « qui propose d'entamer un travail sérieux d'éducation dense et libériste au sein de l'élément féminin, afin de mieux réaliser le cycle préparatoire des revendications sociales » ; elle annonce la poursuite de la bataille contre la prostitution, contre la « camorra » et l'obscurantisme, ainsi que sa volonté de créer « les consciences pour compenser cette société hypocrite qui considère encore la femme comme un jouet de plaisir » (Legati 1913b, 1). 
Journalistes et revues féministes anarchistes en Italie au début du XXe siècle

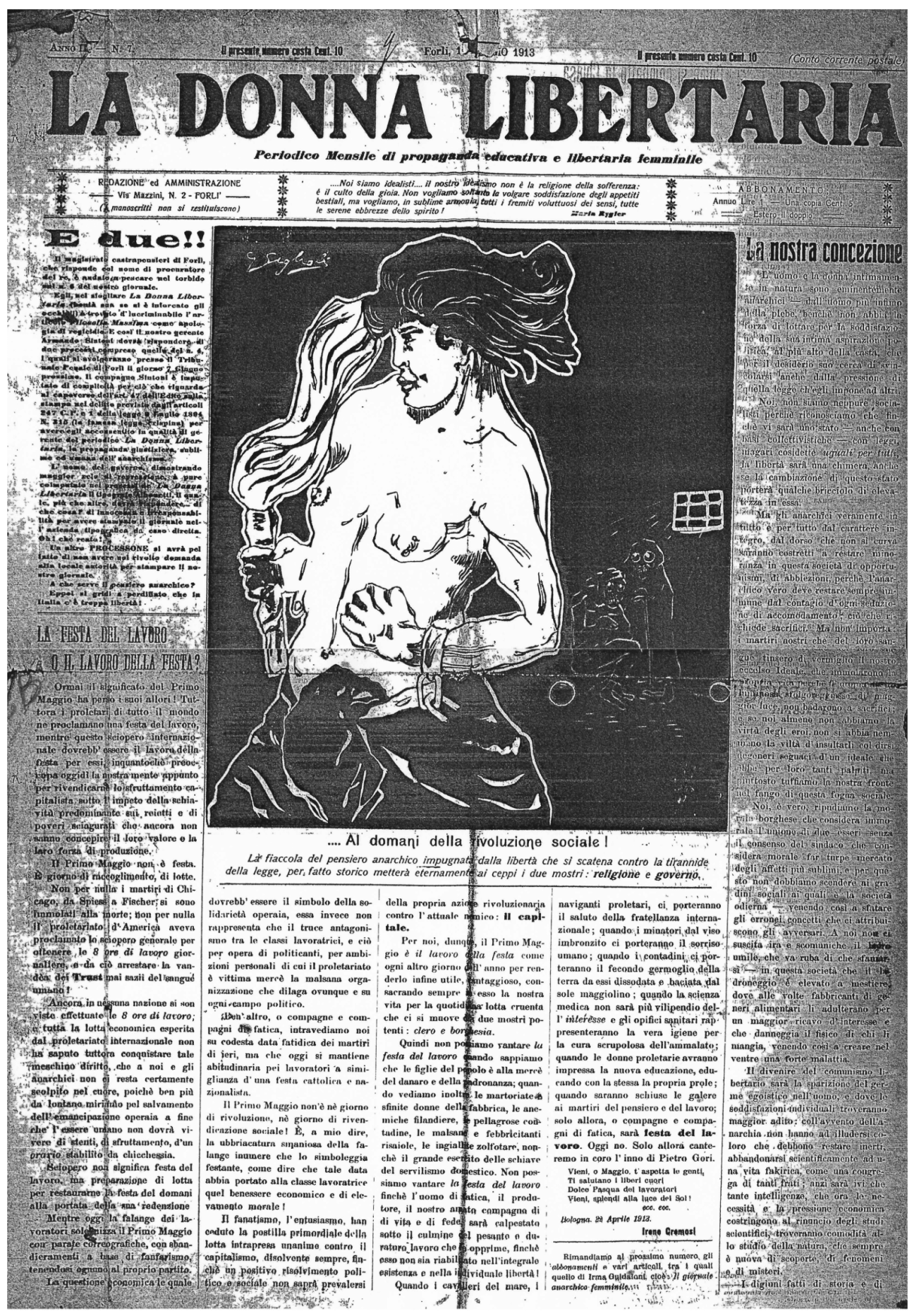

FiguRE 3 - Première de couverture dę La Donna libertaria, $\mathrm{n}^{\circ}$ 7, Forlì, 1er mai 1913 (Biblioteca Libertaria Armando Borghi, Castel Bolognese, Italie) 
Journalistes et revues féministes anarchistes en Italie au début du XXe siècle

La seconde édition du journal continue la campagne antimilitariste et développe d'autres thèmes classiques de la propagande anarchiste, comme l'anticléricalisme (Imondi 1913, 2; Dervisi 1913a, 2, 1913b, 3), l'antialcoolisme (« Tribuna antialcoolista »1913, 2; antialcoolista 1913, 3), la critique du mariage bourgeois en faveur de " l'amour libre »- dans des articles généralement signés par des noms de plume masculins (Romiti 1912, 2; Ruscello 1913, 3 ). On constate cependant un intérêt du journal pour des thèmes propres au monde féminin, comme la bataille contre la prostitution (Montagna 1912, 2 ; Ruscello 1912b, 3-4; Tonetti 1913, 3) et la traite des enfants (Mori 1912, 4), l'éducation des mères prolétaires (Legati 1913d, 2), la famille et la maternité. Dans le dernier numéro, Irma Guidaloni explique que ces thèmes ont rarement été traités dans les autres périodiques anarchistes, « car ceux-ci mènent leur mission supérieure de propagande au sein de la classe ouvrière ». La Donna libertaria entend combler ces manques et œuvrer à l'élévation des femmes afin qu'elles cessent d'être des victimes :

Sans la femme émancipée, ni les partis politiques ni les penseurs de l'anarchisme ne pourront jamais atteindre l'objectif d'amélioration sociale, encore moins, sans les femmes, l'action révolutionnaire ne pourra être menée afin de renverser le régime bourgeois actuel. (Guidaloni 1913b, 1)

Elle souhaite que les femmes apprennent à lutter aux côtés des hommes, et qu'elles comprennent que leur émancipation est « la défense de la vie ». La Donna libertaria avance en particulier des propositions originales sur la liberté de la maternité et le féminisme.

\section{Liberté de maternité et rôle des mères}

Alors qu'en Italie très peu de femmes interviennent dans les débats sur la liberté de la maternité et la contraception, et que le mouvement néomalthusien est quasi exclusivement monopolisé par les hommes ${ }^{13}$, La Donna libertaria publie plusieurs articles ayant trait au contrôle des naissances, en prenant ouvertement la défense du néomalthusianisme. Les anarchistes avaient déjà commencé à divulguer des théories et pratiques de la « procréation consciente »

13. On le constate en parcourant les noms des auteurs de plus d'une centaine de titres sur l'éducation sexuelle (Rifelli et Ziglio 2006). Voir aussi Loconsole (2017). 
Journalistes et revues féministes anarchistes en Italie au début du XXe siècle

auprès de la classe ouvrière italienne (De Longis 1982; Masjuan 2002) : en 1904, Il Pensiero avait publié des textes d'anarchistes français (comme la conférence de Sébastien Faure, Le Problème de la population, et des articles de Paul Robin et de Nelly Roussel ${ }^{14}$ ), récupérant la « loi de Malthus », selon laquelle la progression de la population, plus rapide que celle des subsistances, ne pouvait conduire qu'à une catastrophe si on ne mettait pas un terme à la croissance démographique. Suite au congrès de Florence de 1910 consacré à la question sexuelle, Luigi Berta ${ }^{15}$ fonde à Turin en 1913 la Ligue néomalthusienne italienne (LNI) et prend la défense de l'ouvrier anarchiste Secondo Giorni, auteur d'un manuel de procédés anticonceptionnels pour le prolétariat, accusé d'outrage à la pudeur (Giorni 1911).

Le premier texte qui aborde le sujet dans La Donna libertaria est signé Libero Gentile : l'auteur, tout en se montrant très sceptique quant au bien-fondé de la théorie de Malthus, invite les lectrices du journal à étudier la question et à écouter les conseils de Secondo Giorni. Non seulement la contraception permet d'éviter la naissance de « dégénérés » en améliorant l'espèce ${ }^{16}$, mais elle agirait aussi comme "prophylaxie sociale », pour diminuer la quantité de misérables. Gentile insiste néanmoins sur le fait que la limitation des naissances ne doit jamais être imposée mais doit être librement acceptée. Dans le numéro suivant, "l'Amica di Tomasina » va plus loin, et formule l'idée de « grève des ventres » comme refus du capitalisme :

Vous, mères prolétariennes, qui nous avez mis au monde pour la joie et non pour la tristesse, pour l'entente et non pour la discorde, pour l'amour et non pour la haine, pour le travail et non pour le carnage, répondez en cessant d'être des génitrices passives et résignées. Refusez à la société capitaliste les victimes qu'elle réclame avec insistance, car elle ne tend qu'à assassiner vos enfants. Pauvres mères, chères mères, mères prolétariennes cessez de faire des victimes! (Tomasina 1913)

14. Sur les néomalthusiens français, voir Cova (2011).

15. Médecin turinois, auteur de l'ouvrage pratique Per limitare la prole (1913) et directeur de la revue L'Educazione sessuale (1913-1915) dont l'objectif est de faire de la publicité aux théories néomalthusiennes.

16. « Les maladies autant que les privations, les vices comme les défauts, se transmettent de génération en génération, se multiplient toujours au détriment de l'espèce et compliquent de plus en plus le problème de la justice et de la liberté » (Gentile 1912, 3). 
Journalistes et revues féministes anarchistes en Italie au début du XXe siècle

Le numéro d'après publie une accusation contre les maris « assassins » qui infligent des grossesses et des accouchements répétés à leur femme, allant jusqu'à causer leur mort anticipée (Neo-Malthusianista 1913, 2). Irene Cromosi revient encore une fois sur le sujet, en expliquant que le refus d'avoir une progéniture est un choix de vie qui mérite d'être défendu au nom de l'émancipation féminine : « La femme, qui représente encore le sexe faible, devrait concevoir que vivre sans progéniture est un choix de vie émancipé, combatif et économiquement et politiquement heureux, face à une société qui réclame sans relâche de nouvelles forces humaines actives et travailleuses » (Cromosi 1913, 1).

La Donna libertaria invite ses lectrices à prendre connaissance des ouvrages néomalthusiens, non seulement celui de Secondo Giorni, mais aussi celui de la docteure Ettorina Cecchi (1913) et d'autres, qui selon Cromosi ne doivent pas être considérés comme immoraux. Le propos est renforcé par une lettre envoyée à la rédaction, qui prend la défense des femmes infanticides et accuse à leur place la société qui n'offre aucun soutien aux femmes misérables et invoque à la place le dogme de la « repopulation »: 《elles ne devraient pas être traitées de criminelles, mais plutôt la société qui les met dans la quasiobligation de détruire le fruit de leur maternité non désirée » (Femminista 1913, 1).

On voit ainsi que La Donna libertaria va très loin dans ses propositions et ses réflexions sur la liberté de maternité, même si le contrôle des naissances est davantage considéré comme un remède à la misère que comme un moyen d'amélioration de la condition féminine. Maria Rygier elle-même se montre plus féministe, en imaginant une société où les intérêts des femmes seraient pris en compte :

je crois que dans toute société, même dans la société libre de l'avenir, la procréation prudente sera pratiquée au nom des intérêts suprêmes de la femme, qui voudra être mère de quelques enfants forts, mais ne s'adaptera pas à passer les meilleures années de sa vie dans une succession continue de grossesses et d'allaitement. (Rygier 1914).

La Donna libertaria ne manque pas par ailleurs de célébrer le rôle des mères dans la lutte révolutionnaire anarchiste, et les invite de façon répétée à agir tout particulièrement contre le militarisme. Par exemple, la « bonne mère » 
Journalistes et revues féministes anarchistes en Italie au début du XXe siècle

anarchiste doit être capable de résister aux stéréotypes de genre qui l'amènent à élever différemment les filles et les garçons. Au lieu de surprotéger les filles et de se réjouir de la «virilité » de leurs garçons habillés en militaires et s'enivrant au bar, les mères qui « aiment vraiment intensément leurs fils et leurs filles » doivent se libérer des préjugés et en particulier elles doivent craindre : « la guerre, les expéditions coloniales, les massacres de femmes, d'enfants, de personnes âgées et de malades, l'oisiveté et le vice dans les casernes, les maladies dites vénériennes, les fièvres typhoïdes et l'alcoolisme pour leurs enfants » (Parigina 1913, 4).

Le dernier numéro publie un récit qui met en scène un militaire tué dans les guerres coloniales, qui dans son dernier souffle demande pardon à sa mère d'être devenu un assassin (Gabella 1913, 2). La revue rejoint ainsi les positions des leaders du mouvement d'émancipation des femmes, qui voyaient dans la « maternité forte » le tremplin pour modifier les inégalités de la condition féminine (D'Amelia 2005), mais elle refuse le culte de la mère sacrificielle, la mater dolorosa, fidèle au programme anarchiste antimilitariste et antinationaliste. La Donna libertaria condamne en effet le patriotisme (Perini 1912, $3)$.

\section{La lutte contre l'antiféminisme}

L'engagement de La Donna libertaria contre le militarisme et son soutien aux victimes de la répression anti-anarchiste lui vaut d'être régulièrement séquestrée par l'autorité judiciaire, comme le signalent les rédactrices (Dervisi, Rossi, et Guidaloni 1913c, 1, 1913a, 1, 1913b, 1), et il est probable qu'elle ait été définitivement supprimée par les tribunaux après le numéro 8 . Cependant cette initiative ne fut pas particulièrement soutenue ni défendue par les milieux anarchistes. En effet, les femmes journalistes doivent se battre sur plusieurs front, y compris au sein du mouvement anarchiste, contre les compagnons qui ne voient pas d'un bon oeil un journal géré par des femmes.

Par exemple, Tomasina se défend des moqueries de « Bibi » contre les femmes journalistes, publiées dans les pages de L'Agitatore (Tomasina 1912a, 4), et Irma Guidaloni se plaint de l'antiféminisme de certains anarchistes : «J'ai vu plusieurs publications anarchistes que je juge opposées au féminisme en général, en particulier à sa partie intelligente et intellectuelle » (Guidaloni 1913a, 3). 
Journalistes et revues féministes anarchistes en Italie au début du XXe siècle

La revue subit en particulier les attaques de Domenico Zavattero, en conflit également avec Maria Rygier, car il n'avait pas accepté de devoir lui céder la direction de L'Agitatore. Les rédactrices ne mâchent pas leurs mots pour se défendre contre les articles qu'il publie dans Le Canaglie rosse, supplément de La Barricata, où il insinue que les rédactrices de La Donna libertaria étaient en réalité des hommes déguisés en femmes, en les invitant à plus de sérieux (Cantarelli 1980, 251-52). Irma Guidaloni lui lance « vous ne méritez pas le droit de rester dans le mouvement anarchiste » (Guidaloni 1913c, 4); puis Amelia Legati déclare avec encore plus de virulence qu'il est une « canaille matriculée » et un « vulgaire diffamateur » (Legati 1913c, 4), en même temps qu'elle confirme son appartenance au sexe féminin.

Après la cessation de la revue, les écrivaines anarchistes donnent vie à de nouvelles publications périodiques gérées en couple : Rafanelli et Monanni fondent la revue antimilitariste milanaise La Libertà (1913-1915), tandis que Nella Giacomelli participe à Volontà (1913-1915), publié à Ancône, où elle évoque notamment le chemin qu'il reste à parcourir face au plus grand des préjugés, à savoir l'antiféminisme,

qui caractérise si étrangement les opinions de la majorité des hommes. Car en théorie, nous admettons, oui, l'égalité en matière de droits civils et de capacités morales des deux sexes, en dehors de leurs fonctions spécifiques, mais nous ne savons cependant pas comment échapper à une espèce d'arrogance moqueuse devant une femme qui adopte des attitudes d'indépendance. (Giacomelli (Petit-Jardin) 1913)

Un second et dernier périodique féminin anarchiste voit le jour à Pontremoli, L'Alba libertaria, periodico mensile di propaganda femminile anarchica, en pleine période de propagande interventionniste en Italie ${ }^{17}$. Il publie quatre numéros entre le 15 février et le 16 mai 1915, sous la responsabilité éditoriale de Giovanni Romiti. Les noms des membres du comité de rédaction ne sont pas indiqués, mais un des articles du premier numéro est signé Irma, très

17. Je tiens à remercier la bibliothèque de l'International institute of social history d'Amsterdam, qui m'a envoyé gracieusement les reproductions des numéros de la revue dans le contexte de la crise sanitaire où l'accès direct à ses collections était limité. 
Journalistes et revues féministes anarchistes en Italie au début du XXe siècle

vraisemblablement Irma Pagliai ${ }^{18}$. Si la plupart des textes est anonyme, on trouve aussi quelques signatures d'écrivaines anarchistes connues comme Priscilla Fontana, Leda Rafanelli et Nazzarena Diamanti ${ }^{19}$. Selon Elena Bignami, « il s'agit de la dernière tentative désespérée pour "éduquer" les femmes en général aux principes anarchistes et en particulier, à présent, à l'antimilitarisme et à l'opposition à la guerre, comme les hommes n'étaient pas parvenus à le faire » (Bignami 2016, 81).

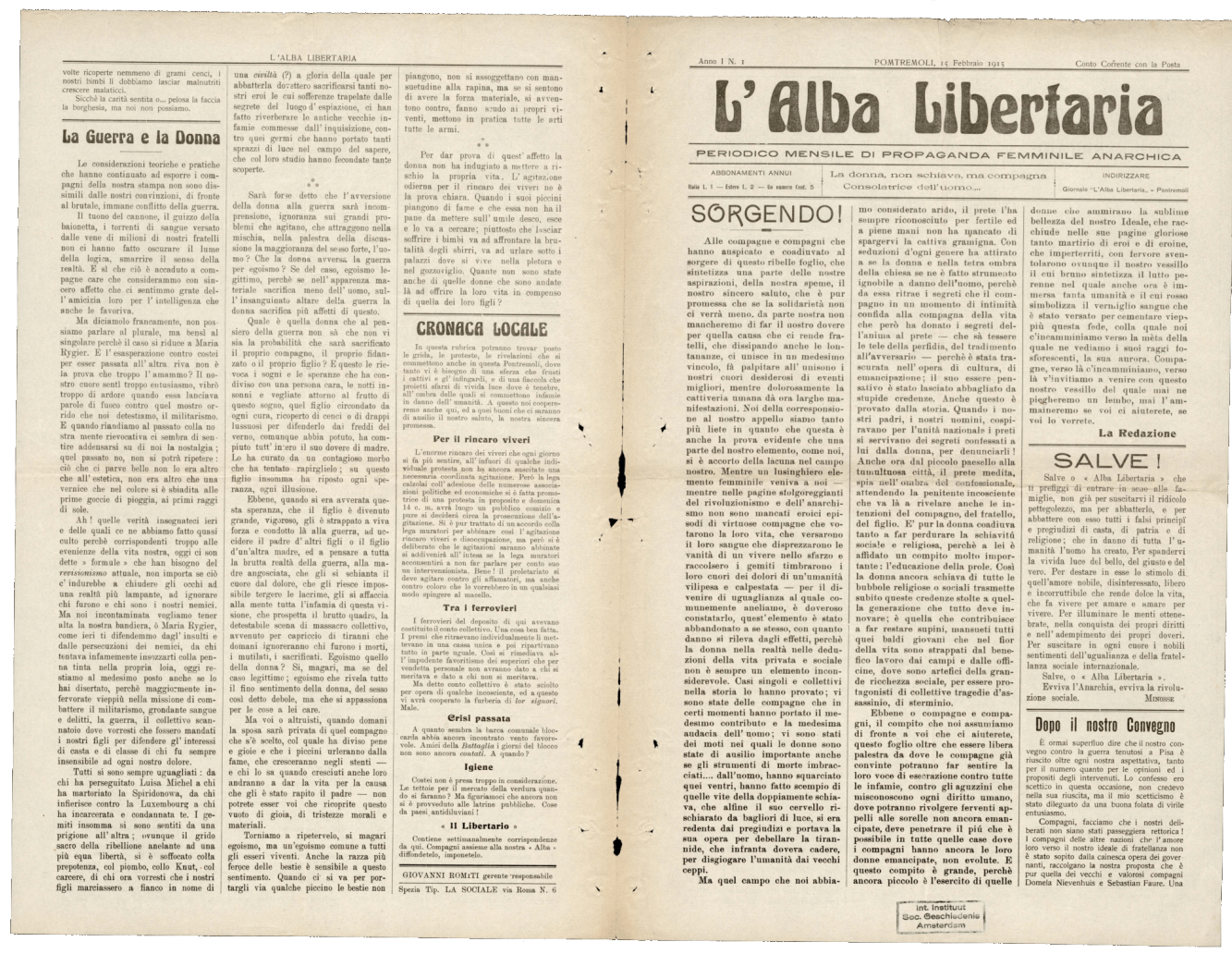

Figure 4 - Première de couverture de L'Alba libertaria, n ${ }^{\circ} 1$, Pontremoli, 15 février 1915 (International institute of social history collections, Amsterdam, Pays-Bas)

18. Irma Pagliai est née à Pontremoli en 1893 dans une famille anarchiste; employée dans les chemins de fer, elle participe activement à la propagande antimilitariste et au groupe féminin libertaire de Pontremoli (Bignami 2016, 82).

19. Une ouvrière textile qui prend la défense des tisseuses de l'usine Merlini de Vergato (Bologne), spécialisée dans la toile de jute (Diamanti 1915, 4). 
Journalistes et revues féministes anarchistes en Italie au début du XXe siècle

La revue publie de dures attaques contre les « mères inconscientes » qui envoient leurs fils à la mort (Fontana 1915, 2-3; « La guerra e la donna »1915, 4 ; Irma 1915, 1-2), mais aussi contre les anarchistes qui n'ont pas éduqué leurs compagnes à la politique. En particulier, «Emma» de Turin rappelle que les théories anarchistes prônent la « complète émancipation de cette compagne de l'homme ", mais que dans la pratique « bien peu d'anarchistes considèrent la femme comme leur compagne naturelle ». C'est " leur rôle de l'éduquer moralement et intellectuellement pour qu'elle abandonne les préjugés incarnés actuellement dans ses viscères et qu'elle vienne lutter pour l'idée de rédemption sociale » : « Ce serait un grand pas pour notre cause et surtout nous aurions évité de voir peut-être demain des obstacles sur notre route ou de recevoir d'une femme une possible trahison » (Emma 1915, 1).

La revue réagit notamment à la 《trahison » de Maria Rygier, l'icône de l'antimilitarisme qui a rejoint le camp des interventionnistes. Malgré les dernières tentatives pour résister aux forces militaristes (Rafanelli 1915), l'entrée en guerre de l'Italie et les déplacements constants des militants anarchistes entrainent des ruptures au sein des couples et dans leurs relations amicales, et le second périodique féminin anarchiste cesse lui aussi ses publications.

En conclusion, la participation des femmes écrivaines à la presse anarchiste en Italie au début du $\mathrm{XX}^{\mathrm{e}}$ siècle est loin d'être anecdotique. De nombreux journaux sont fondés par des couples, où les militantes font entendre régulièrement leur voix dans la propagande antimilitariste mais aussi pour la dignité et la liberté des femmes. C'est grâce à l'activité et à la plume des anarchistes Zelmira Binazzi, Aida Latini, Nella Giacomelli, Leda Rafanelli, Fanny Dal Ry, Francesca Aondio, Priscilla Poggi et sa fille Jessa Fontana, Irma Pagliai, auxquelles il faut ajouter celles se cachant derrière des pseudonymes et les nombreuses anonymes, que les idées anarchistes ont pu être diffusées et discutées plus largement. Parmi leurs initiatives les plus remarquables, la revue $L a$ Donna libertaria se distingue à plusieurs titres des journaux féminins de son époque et également des journaux anarchistes rédigés par des hommes : première revue anarchiste féminine, qui se propose de diffuser la pensée libertaire parmi le lectorat féminin, elle développe des thèmes qui sont partagés par d'autres journaux du milieu, en particulier celui de l'antimilitarisme, alors qu'une majorité des journaux féministes abandonna l'engagement pacifiste après la conquête de la Libye en 1911. 
Journalistes et revues féministes anarchistes en Italie au début du XXe siècle

La Donna libertaria, puis le second périodique féminin anarchiste L'Alba libertaria, accusent en même temps les compagnons anarchistes d'avoir négligé l'élément féminin au sein du mouvement et avancent des propositions originales pour l'émancipation des femmes, notamment la « grève des ventres 》 et l'éducation des mères prolétaires au refus de la guerre. On peut donc saluer le courage des rédactrices qui eurent à se battre contre les préjugés et les attaques de tous bords et insérer ces titres de plein droit parmi les journaux féministes.

\section{Bibliographie}

antialcoolista, L'operaia. 1913. «Tribuna antialcoolista ». La Donna libertaria, n 5 (Mars).

Antonioli, Maurizio. 2002. « Alla ricerca dello pseudonimo perduto. Per il DBAI ». Rivista Storica dell'Anarchismo IX (1) : n.p. http://bibliotecaborghi. org/wp/wp-content/uploads/2015/12/antonioli_pseudonimi.pdf.

- éd. 2003. Dizionario biografico degli anarchici italiani. Pisa : BFS.

Antonioli, Maurizio, et Pier Carlo Masini. 1999. Il sol dell'avvenire : l'anarchismo in Italia dalle origini alla prima guerra mondiale. Biblioteca di storia dell'anarchismo 7. Pisa : BFS.

Aondio, Francesca. 1907. « Alle donne ». La Protesta umana, août.

_. 1909. «Protesta ». La Protesta umana, avril.

Bartoloni, Stefania. 2017. Donne di fronte alla guerra : pace, diritti e democrazia (1878-1918). Quadrante Laterza 211. Bari : GLF editori Laterza.

Berta, Luigi. 1913. Per limitare la prole : i mezzi migliori per prevenire la gravidanza. Torino : L'educazione sessuale.

Berti, Giampietro D. 2003. Errico Malatesta e il movimento anarchico italiano e internazionale : 1872-1932. Saggi di storia 15. Milano : F. Angeli.

Bignami, Elena. 2011. Le schiave degli schiavi : la "questione femminile" dal socialismo utopistico all'anarchismo italiano, 1825-1917. Saggi e monografie del Dipartimento di discipline storiche, geografiche e antropologiche dell'Università di Bologna, nuova ser., 1. Bologna : CLUEB. 
Journalistes et revues féministes anarchistes en Italie au début du XXe siècle

— 2016. «"Se le guerre le facessero le donne". L'opposizione delle anarchiche italiane alla guerra (1903-1915) ». DEP Deportate, esuli, profughe XII (31) : 54-85.

-, éd. 2018. Le donne nel movimento anarchico italiano : 1871-1956. Eterotopie, n. 451. Milano : Mimesis.

Buttafuoco, Annarita. 1988. Cronache femminili : temi e momenti della stampa emancipazionista in Italia dall'Unità al Fascismo. Arezzo : Dipartimento di studi storico-sociali e filosofici Universita degli Studi di Siena.

Cantarelli, Rossella. 1980. « La donna libertaria, Parma 1912-Forlì 1913 : considerazioni su un giornale anarchico femminile italiano ». In Alle origini del Pci. Atti del Convegno su Gastone Sozzi, 237-52. Cesena : Circolo Antonio Gramsci.

Cecchi, Ettorina. 1913. Neo-malthusianismo pratico : anatomia e fisiologia degli organi genitali, fenomeno della generazione, mezzi scientifici e pratici per evitare la gravidanza. Bologna : La controcorrente.

Chessa, Fiamma, éd. 2008. Leda Rafanelli : tra letteratura e anarchia. Reggio Emilia : Biblioteca Panizzi.

Colaone, Sara, Francesco Satta, et De SantisLuca. 2016. Leda : che solo amore e luce ha per confine. Roma : Coconino press : Fandango.

Corabi, Gilda. 2012. « Scrittrici dell'Ottocento ». In Atlante della letteratura italiana, vol. 3 Dal romanticismo a oggi, édité par Domenico Scarpa, 162-76. Torino : Einaudi.

Cova, Anne. 2011. Féminismes et néo-malthusianismes sous la IIIe République, la liberté de la maternité. Logiques sociales. Paris : Harmattan.

Cromosi, Irene. 1913. « Prolisti e neo-malthusiani ». La Donna libertaria, n 6 (Avril).

Dal Ry, Fanny. 1920. Giù le armi. Genova : Lib. Ed. La Pace.

D'Amelia, Marina. 2005. La mamma. L'identità italiana 41. Bologna : Il mulino.

De Longis, Rosanna. 1982. "In difesa della donna e della razza ». Nuova Donna Woman Femme, n 21 : 149-77. 
Journalistes et revues féministes anarchistes en Italie au début du XXe siècle

Dervisi, Adele. 1913a. « Come si combatte la Chiesa ». La Donna libertaria, n 7 (Mai).

_. 1913b. «Il prete ». La Donna libertaria, n 8 (Juin).

Dervisi, Adele, Marzia Rossi, et Irma Guidaloni. 1913a. «E due ». La Donna libertaria, n 6 (Avril).

—. 1913b. «E tre ». La Donna libertaria, n 8 (Juin).

—. 1913c. «E uno ». La Donna libertaria, n 5 (Mars).

Diamanti, Nazzarena. 1915. "Alle operaie addette al Jutificio Merlini ». L'Alba libertaria, n 2 (Avril).

Emma. 1915. «L'emancipazione della donna ». L'Alba libertaria, n 4 (mai).

Femminista. 1913. « Maternità non desiderata ». La Donna libertaria, n 6 (Avril).

Fontana, Priscilla. 1915. " Alle madri incoscienti ». L'Alba libertaria, n 1 (Février).

Frattini, Claudia. 2008. Il primo Congresso delle donne italiane, Roma 1908: opinione pubblica e femminismo. Novecento periodico 2. Roma : Biblink.

Gabella, Ermete. 1913. « Mamma! Mamma!» La Donna libertaria, n 8 (Juin).

Gazzetta, Liviana. 2018. Orizzonti nuovi : storia del primo femminismo in Italia (1865-1925). Storia delle donne e di genere; Singolare 9. Roma : Viella.

Gentile, Libero. 1912. « Malthus e neo-malthusiani ». La Donna libertaria, n 3 (Décembre).

Giacomelli, Nella. 1902. « Contro il pregiudizio ». Il Grido della folla, juillet. _. 1903a. "Una buona battaglia. Amore unico o multiplo ». Il Grido della folla, juin.

—. 1903b. «Un'agressione per libero amore ». Il Grido della folla, septembre.

Giacomelli (Petit-Jardin), Nella. 1913. « Noticina... femminista ». Volontà, décembre. 
Journalistes et revues féministes anarchistes en Italie au début du XXe siècle

Giacomini, Ruggero. 1990. Antimilitarismo e pacifismo nel primo Novecento : Ezio Bartalini e La Pace, 1903-1915. Milano : F. Angeli.

Giorni, Secondo. 1911. L'arte di non far figli : neomalthusianismo pratico. Firenze : Tip. Cooperativa.

Giulietti, Fabrizio. 2012. Storia degli anarchici italiani in età giolittiana. Studi e ricerche storiche 404. Milano : F. Angeli.

Guidaloni, Irma. 1913a. « Guerra alla donna ». La Donna libertaria, n 4 (Février).

—. 1913b. «Il giornale anarchico femminile ». La Donna libertaria, n 8 (Juin).

—. 1913c. « Risposta... ad una domanda ». La Donna libertaria, n 8 (Juin).

Guidoni, Christiane. 1994. « Leda Rafanelli : Donna e Femmina ». Chroniques italiennes, n 39/40:63-73.

《 Il nostro processo in tribunale ». 1913. La Donna libertaria, n 8 (Juin).

Imondi, G. 1913. «A Dio ». La Donna libertaria, n 5 (Mars).

Irma. 1915. « La donna e l'intervenzionismo ». L'Alba libertaria, n 3 (Avril). « La guerra e la donna ». 1915. L'Alba libertaria, n 1 (février).

Legati, Amelia. 1913a. "Abbasso la guerra! » La Donna libertaria, n 5 (Mars).

—. 1913b. « Continuando... ». La Donna libertaria, n 4 (Février).

_. 1913c. « Dichiarazione personale ». La Donna libertaria, n 8 (Juin).

—. 1913d. « La scuola per le mamme proletarie ». La Donna libertaria, n 6 (Avril).

Loconsole, Matteo. 2017. Storia della contraccezione in Italia tra falsi moralisti, scienziati e sessisti. Bologna : Pendragon.

M., M. 1907. « Conquistiamo la donna ». La Protesta umana, décembre.

Mangani, Lidia. 1989. « Fanny Dal Ry. Una maestra elementare tra femminismo e pacifismo ». Storia e problemi contemporanei 2 (4) : 87-107. 
Journalistes et revues féministes anarchistes en Italie au début du XXe siècle

Masini, Pier Carlo. 1973. « Le due Pasionarie dell'anarchia italiana ». Storia illustrata, n 191 : 119-28.

Masjuan, Eduard. 2002. "Neo-malthusianesimo e anarchia in Italia : un capitolo della storia dell'ecologismo dei poveri? » Meridiana, n 44 : 195-222.

Mauri, Antonella. 2011. «Entre colonialisme et métissage culturel : Leda Rafanelli, anarchiste et musulmane ». In Oublier les colonies. Contacts culturels hérités du fait colonial, édité par Isabelle Felici, Jean-Charles Vegliante, et André Ughetto, 177-95. Paris : Mare \& Martin.

Montagna, Eliseo. 1912. « La prostituzione ». La Donna libertaria, n 1 (Octobre).

Montesi, Barbara. 2013. Un "anarchica monarchica» : vita di Maria Rygier (1885-1953). Il presente come storia, nuova serie, 8. Napoli : Edizioni scientifiche italiane.

Mori, Ida. 1912. " La tratta dei fanciulli ». La Donna libertaria, n 2 (Novembre).

Neo-Malthusianista, Marianna. 1913. «Premio a l'assassinio ». La Donna libertaria, n 4 (Février).

Ongaro, Ercole. 2019. Nella Giacomelli : un'anarchica controcorrente. Collana Biografie. Milano : Zero in condotta.

Parigina, La. 1913. "L'amor materno ». La Donna libertaria, n 6 (Avril).

Perini. 1912. « Amor patrio ». La Donna libertaria, n 3 (Décembre).

Pisano, Laura, éd. 2004. Donne del giornalismo italiano : da Eleonora Fonseca Pimentel a Ilaria Alpi : dizionario storico bio-bibliografico, secoli XVIII-XX. Studi e ricerche di storia dell'editoria 24. Milano : F. Angeli.

Rafanelli, Leda. 1904. «Il Femminismo ». Il Pensiero, septembre.

_. 1906. «Donne, aiutateci! » Energia!, avril.

—. 1915. Abbasso la guerra! Milano : Soc. Ed. Milanese.

Rifelli, Giorgio, et Corrado Ziglio. 2006. Per una storia dell'educazione sessuale, 1870-1920. 2e éd. Azzano San Paolo: Junior. 
Journalistes et revues féministes anarchistes en Italie au début du XXe siècle

Romiti, Giovanni. 1912. "Gli anarchici e l'amore ». La Donna libertaria, n 3 (Décembre).

Ruscello, Eliseo. 1912a. " Chi sono gli anarchici? » La Donna libertaria, n 2 (Novembre).

(Novembre).

—. 1913. « Del libero amore ». La Donna libertaria, n 5 (Mars).

Rygier, Maria. 1912. «Una nobile lettera di Maria Rygier ». La Donna libertaria, n 1 (Octobre).

—. 1914. «Il neomalthusianismo è immorale? » L'Educazione sessuale, n 9 (janvier) : 8 .

Sacchetti, Giorgio. 1983. Sovversivi in Toscana : 1900-1919. Todi : Altre edizioni.

Scriboni, Mirella, éd. 2008. Abbasso la guerra! Voci di donne da Adua al primo conflitto mondiale, 1896-1915. Cultura storica 33. Pisa : BFS.

Tagliaventi, Simona. 2014. " Socialista, femminista, antimilitarista. Fanny Dal Ry ». In Donne nella grande guerra, 193-205. Bologna : Il mulino.

Tomasina. 1912a. " A "Bibi" de "L'Agitatore" ». La Donna libertaria, n 3 (Décembre).

—. 1912b. «Alle donne proletarie ». La Donna libertaria, n 3 (Décembre).

_. 1912c. « La donna libertaria! » La Donna libertaria, n 1 (Octobre). —. 1912d. « Siamo violenti? » La Donna libertaria, n 2 (Novembre).

Tomasina, L'Amica di. 1913. « Per il Neo-malthusianismo ». La Donna libertaria, n 4 (Février).

Tonetti, O. 1913. « La prostituta ». La Donna libertaria, n 8 (Juin).

« Tribuna antialcoolista ». 1913. La Donna libertaria, n 7 (Mai). 